\title{
Abordaje Quirúrgico de un Canino Maxilar Impactado en Posición Vestibular para Tracción Ortodóncica: Reporte de Caso y Revisión de la Literatura
}

\author{
Surgical Approach of Vestibular Position Impacted Maxillary Canine for Orthodontic \\ Traction: Case Report and Literature Review
}

\author{
Víctor Javier Beltrán Varas, ${ }^{* * *}$; Pedro Flores Bengoechea ${ }^{* * *}$; Nerilda García Alarcón ${ }^{* * * *}$; \\ Mario Cantín* \& Ramón Fuentes Fernández*
}

BELTRÁN, V. V. J.; FLORES, B. P.; GARCÍA, A. N.; CANTÍN, M. \& FUENTES, F. R. Abordaje quirúrgico de canino maxilar incluido en posición vestibular para tracción ortodóncica: reporte de caso y revisión de la literatura. Int. J. Odontostomat., 5(3):220-226, 2011.

RESUMEN: Los dientes impactados presentan muchos problemas. Pueden comprometer la movilidad dentaria, la estética, y los resultados funcionales. Después del tercer molar superior, los caninos superiores presentan la impactación más común, los cuales pueden impactar en posición vestibular o palatina, y son más comunes en mujeres que en varones. Con un manejo terapéutico quirúrgico y ortodoncico adecuado, los caninos superiores impactados pueden erupcionar y ser guíados a una posición adecuada en el arco dental. En este artículo se presenta un caso quirúrgico-ortodóncico de un canino superior impactado vestibular en una mujer adulta, tratada mediante la técnica cerrada con preservación de las papilas.

PALABRAS CLAVE: Canino maxilar impactado; Técnicas quirúrgica; Técnicas de ortodoncia.

\section{INTRODUCCIÓN}

El diagnóstico precoz, la intercepción oportuna y un tratamiento multidisciplinario apropiado hacen posible que un canino maxilar impactado pueda ser reposicionado en el lugar que le corresponde dentro del arco dentario, de ahí la importancia que los clínicos le han dado a este tema, debido a que es el diente con mayor frecuencia de impactación seguido por los terceros molares (Bishara, 1992; Ngan et al., 2005). Otros autores lo reportan en segundo lugar después del tercer molar mandibular (Mcsherry, 1998), de los cuales dos tercios se presentan en posición palatina y un tercio en la zona vestibular (Ericson \& Kurol, 1988). El $8 \%$ de todos los pacientes que padecen impactación de caninos maxilares es de tipo bilateral (Bishara).

Mucho tiempo se especuló que los caninos maxilares tenían un trayecto más tortuoso que cual- quier otro diente para su erupción y que esta situación estaba relacionada con tres factores: el tiempo requerido para su desarrollo, la posición cambiante durante su desarrollo y la distancia que recorre en su vía de erupción. Teorías actuales apuntan a que la impactación de los caninos maxilares estaría relacionada a la ausencia o deformidad de la raíz del incisivo lateral (Becker, 2007) o a condicionantes genéticas (Peck et al., 1994; Baccetti, 1998), sin embargo, ambas teorías están enfocadas al canino impactado en posición palatina.

La erupción ectópica vestibular del canino maxilar está fuertemente asociada con la falta de espacio o el apiñamiento del arco alveolar (Leonardi et al., 2010), y pareciera estar relacionada por largos periodos de erupción, discrepancias del arco dentario maxi-

\footnotetext{
Departamento de Odontología Integral, Facultad de Medicina, Universidad de La Frontera, Chile.

"* Alumno de Programa de Magister en Odontología, Facultad de Medicina, Universidad de La Frontera, Chile.

*.* Especialista en Ortodoncia y Ortopedia Dentomaxilofacial, Hospital Dr. Mauricio Heyermann, Angol, Chile.

***** Alumna Programa de Especialización en Ortodoncia y Ortopedia Dentomaxilofacial, Departamento de Odontología Integral, Universidad de La Frontera, Chile.
} 
BELTRÁN, V. V. J.; FLORES, B. P.; GARCíA, A. N.; CANTíN, M. \& FUENTES, F. R. Abordaje quirúrgico de canino maxilar incluido en posición vestibular para tracción ortodóncica: reporte de caso y revisión de la literatura. Int. J. Odontostomat., 5(3):220-226, 2011.

lar, posiciones anormales, anquilosis, trauma o alteraciones patológicas como quistes entre otras (Rusu et al., 2010). Sin embargo, no hay una claridad total respecto de ésta condición.

Diversas técnicas de aproximación quirúrgica pueden ser utilizadas en su tratamiento, siendo de elección cuando se presenta una posición vestibular del canino maxilar impactado la técnica cerrada o el colgajo desplazado hacia apical, para preservar la inserción gingival (Vanarsdall \& Corn, 1977). La utilización de una de ellas dependerá de la posición vertical del canino impactado.

El propósito de este artículo es presentar una revisión bibliográfica del manejo terapéutico de los caninos maxilares impactados en posiciónvestibular y el reporte de un caso quirúrgico-ortodóncico enfocado a la técnica cerrada con preservación de papilas.

\section{Opciones Terapéuticas}

Tratamiento preventivo. El éxito de esta intervención depende de la edad al momento del diagnóstico y del grado de impactación, siendo importante realizar una evaluación crítica del paciente desde los 10 años de edad, clínica e imagenológicamente (Jacobs, 1992, 1996). Otros autores han propuesto que la extracción de los caninos temporales antes de que el paciente alcance los 11 años de edad podría normalizar la posición de erupción del canino permanente en el $91 \%$ de los casos siempre que la corona se encuentre en posición distal con respecto al eje axial del incisivo lateral (Ericson \& Kurol), sin embargo, el éxito disminuye hasta un $64 \%$ si la corona del canino permanente tiene orientación mesial con respecto al eje descrito anteriormente (Elefteriadis \& Athanasiou, 1996). Leonardi et al. (2004), reportan un $50 \%$ de éxito a 18 meses de realizar exodoncia de los caninos temporales.

Manejo quirúrgico-ortodóncico. Kokich (2004) menciona tres métodos para descubrir un canino maxilar con impactación vestibular: la gingivectomía, la creación de un colgajo en posición apical y la utilización de técnicas de erupción cerrada. Además propuso cuatro criterios para determinar las técnicas correctas para la exposición quirúrgica de un canino superior con impactación vestibular o intraalveolar: la posición vestíbulo lingual de la corona del canino impactado, la posición vertical del diente con respecto a la unión mucogingival, la cantidad de encía en la zona del canino impactado y la posición mesiodistal de la corona del canino.

Vermette et al. (1995) mencionan que para prevenir respuestas indeseables, hay que considerar los siguientes factores: profundidad de la impactación, anatomía de la posición edéntula, velocidad y dirección de la tracción ortodóncica.

El pronóstico del manejo quirúrgico-ortodóncico es bueno y predecible en la mayoría de los casos, dependiendo de una planificación previa exhaustiva en términos de la posición coronal del canino respecto de la tabla vestibular y la calidad del tejido queratinizado (Caccioli et al., 1990).

Tipos de cirugías. Se describen procedimientos cerrados y colgajos desplazados hacia apical para la localización de caninos impactados en posición vestibular, que consisten en localizar el canino retenido a través de un colgajo de espesor total o hacer un desplazamiento apical del colgajo para preservar la banda de encía insertada y bajarlo con fuerza mecánica (Vanarsdall \& Corn). El inconveniente del colgajo de espesor total es que deja una cicatriz en la encía (Macias-Escalada et al., 2005).

La exposición quirúrgica y la extrusión ortodóncica de caninos maxilares impactados consiguen condiciones periodontales clínicamente aceptables; sin embargo, este resultado dependía de la posición vertical y horizontal del canino impactado (Zasciurinskiene et al., 2008).

\section{CASO CLÍNICO}

Paciente sexo femenino de 20 años de edad, sistémicamente sana. Clasificada como braquifacial, clase II esqueletal, discrepancia dentomaxilar negativa severa y canino maxilar derecho impactado (1.3), el cual presentaba una posición oblicua en relación a los ápices del incisivo central y lateral maxilares derechos (1.1 y 1.2) según la imagenología (Fig. 1). A la palpación se encontró aumento de volumen en la zona vestibular superior a la línea muco-gingival por vestibular del incisivo lateral maxilar (1.2), sin embargo, la paciente no relataba dolor o sensibilidad en los dientes en relación a la impactación. Fue sometida a tratamiento previa firma de consentimiento informado.

Se utilizó una técnica quirúrgica cerrada tipo colgajo de espesor total, y el diseño contempló dos descargas, una mesial y distal, con preservación de 


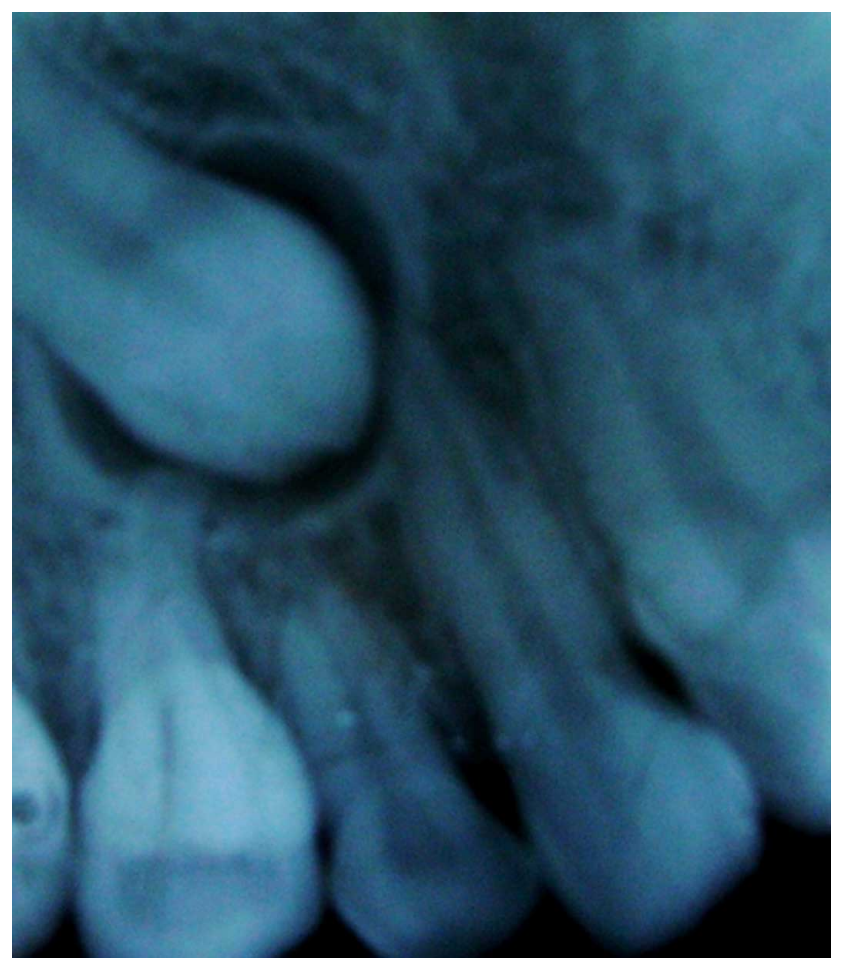

Fig. 1. Radiografía que muestra el nivel de inclusión del canino (1.3) previo a la cirugía. Tiene una dirección oblicua hacia el ápice del incisivo lateral y central (1.2 y 1.1). Nótese la presencia del canino decidual en posición del canino definitivo.

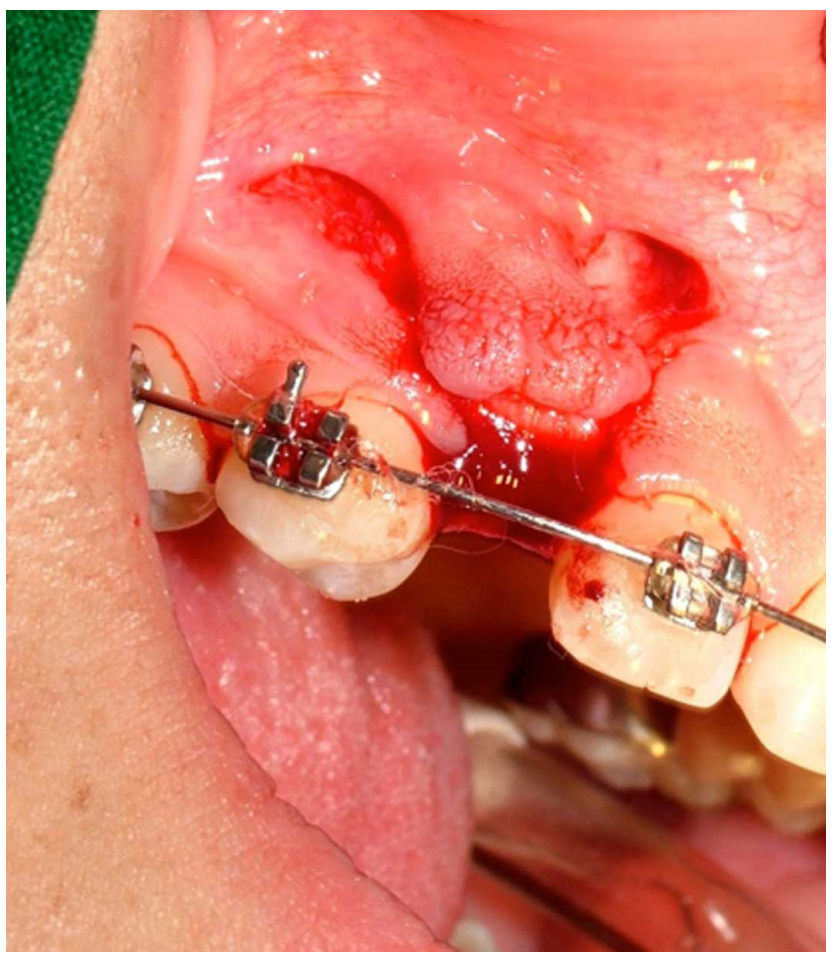

Fig. 2. Colgajo de espesor total con descarga mesial y distal más allá de la línea muco-gingival, respetando las papilas de los dientes vecinos.

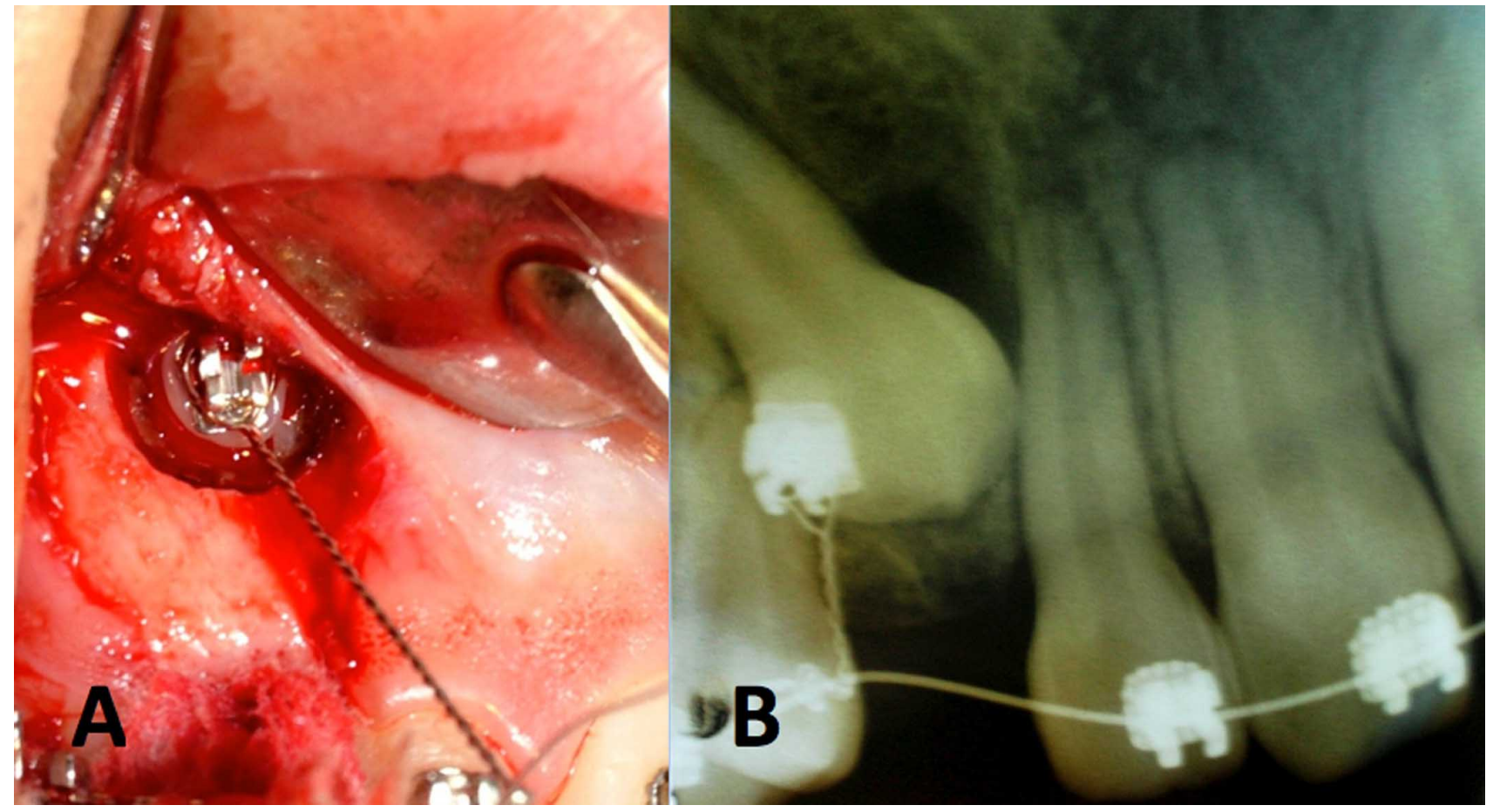

Fig. 3. A. Localización de la porción coronaria del canino maxilar permanente (1.3), luego de realizar una osteotomía controlada de 7 milímetros. Nótese el posicionamiento del bracket. B. Control radiográfico a los 40 días del procedimiento quirúrgico. 
papilas para los dientes vecinos (Fig. 2). Se decidió éste abordaje por el nivel de inclusión del diente y por la facilidad de exposición que podemos lograr con este acceso. Una vez localizado el sitio anatómico en relación a la corona del canino impactado, se decidió realizar una osteotomía controlada con abundante irrigación a través de suero salino. Se utilizó una fresa redonda diamantada de baja velocidad hasta exponer el tejido pericoronario, que fue removido con periostótomo biselado. La exposición se extendió hasta 7 milímetros y se procedió a la cementación de un bracket para tracción en la porción coronaria expuesta del canino.

Una vez realizado el montaje ortodóntico se trabajo el arco superior con arco de nitinol clasico 0.14 (Figs. 3 A y B) y se utilizó un kit adhesivo y resina transbond $\mathrm{XT}, 3 \mathrm{M} 囚$. Para realizar la tracción del canino se unió un alambre de acero 0.8 que queda dentro del colgajo levantado por el cirujano.

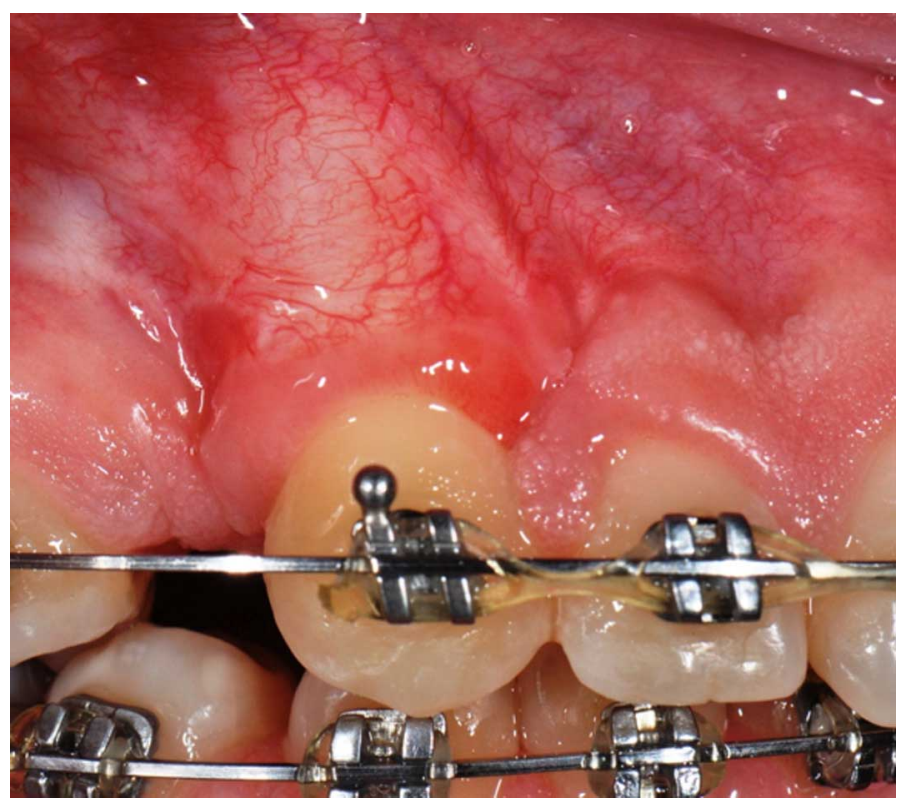

Fig. 4. Visión lateral del canino permanente 5 meses posterior a la tracción ortodóntica-quirúrgica. Nótese la integridad de las papilas mesial y distal al canino permanente después de 5 meses.

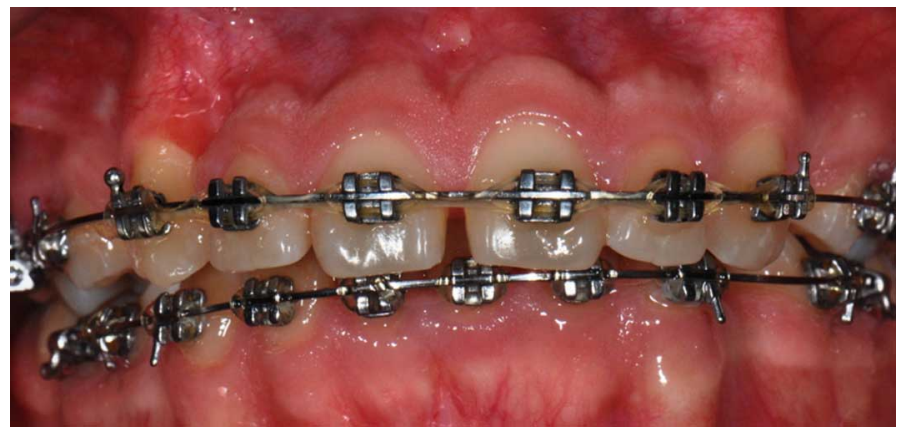

Fig. 5. Visión frontal a los 5 meses de la tracción ortodóncicaquirúrgica.
Después de 5 meses se logró un descenso total del canino maxilar impactado (Fig. 4), con una óptima preservación de papilas (Fig. 5). La posición mesio-distal y la altura ósea crestal de la pieza fue óptima en comparación con las piezas vecinas.

\section{DISCUSIÓN}

El $2 \%$ de la población puede tener caninos superiores impactados, y esta condición es dos veces más frecuente en mujeres que en hombres (Cooke \& Wang, 2006; Proffit et al., 2007). La incidencia a nivel del maxilar es más del doble que en la mandíbula (Yavuz et al., 2007), donde aproximadamente un tercio de los caninos maxilares impactados se encuentran en posición vestibular, y dos tercios se encuentran en posición palatina (Bedoya \& Park, 2009).

La erupción vestibular del canino maxilar está fuertemente asociada con la falta de espacio o el apiñamiento del arco alveolar, largos periodos de erupción, discrepancias del arco dentario maxilar, posiciones anormales, anquilosis, trauma o alteraciones patológicas como quistes entre otras, así como el desplazamiento del folículo dentario, aumento de la presión intravascular dentro del germen dentario, falla en la reabsorción radicular del canino temporal e incluso procesos infecciosos crónicos. Mochizuki et al. (1999), reportan dos casos que relacionan la localización vestibular con la presencia de periodontitis apical crónica del canino temporal, esto sería debido a un proceso infeccioso. A la edad de 8 a 10 años empieza a palparse la prominencia del canino en el fondo del vestíbulo. Los métodos radiográficos más utilizados son las radiografías panorámica, oclusal y periapical, con técnicas de tubo vertical y otras angulaciones. En la actualidad, es de gran ayuda la tomografía computarizada (Pérez et al. 2009).

Si bien el enfoque más conveniente para el manejo de los caninos superiores impactados es el diagnóstico precoz y su intercepción, debido a la escaza prevención de este cuadro es necesario considerar el tratamiento de ortodoncia seguido de la exposición quirúrgica de los caninos para ser llevados a una oclusión normal mediante tracción (Bedoya \& Park). 
El hallazgo de un canino superior incluido e impactado en un paciente adulto representa un manejo terapéutico especial, ya que al ser diagnosticado a edades más avanzadas, a menudo ya han provocado problemas eruptivos, mecánicos, nerviosos o incluso quísticos, lo que justifica su extracción, así como también la falta de espacio en el arco dentario, condicionando la rehabilitación protésica tras la extracción del canino (Magheri et al., 2002).

En nuestro caso, debido a las condiciones locales como ausencia de patología asociada al canino impactado y angulación verticalizada se decidió realizar la cirugía para tracción ortodóntica. Según Ericson \& Kurol la impactación vestibular tiene una angulación vertical más favorable, a diferencia de la impactación palatina que es más horizontal. Se optó por realizar la técnica cerrada mediante un colgajo vestibular de reposición completa junto a la técnica de erupción abierta, la cual consiste en la realización de un colgajo vestibular mucogingival de espesor completo en forma de $U$ teniendo en consideración la preservación de papilas para los dientes vecinos (Cooke \& Wang; Marks et al., 1997). Este tipo de colgajo nos permite igualmente acceder a aquellos dientes supernumerarios, odontomas, etc. en situación vestibular y que dificultan, impiden o bloquean la correcta erupción del diente retenido (Macias-Escalada et al.). Además, la técnica de colgajo vestibular de reposición completa tiene la ventaja de generar la mejor estética gingival y una mayor facilidad de movimiento de los dientes (Kokich; Vermette et al.; Cooke \& Wang).

Según Jacoby (1983) aproximadamente el 85\% de los caninos maxilares impactados palatinos tienen suficiente espacio para la erupción en el arco dentario, mientras que sólo el $17 \%$ de los caninos impactados vestibulares tiene el espacio suficiente. Por esta razón, en nuestro caso, además de la extracción del canino temporal, fue necesaria la realización de un tratamiento ortodóntico para generar el espacio mesio-distal suficiente para posicionar el canino en el arco dentario.

Se ha descrito que aproximadamente en el $80 \%$ de los casos el canino maxilar impacta con el incisivo lateral produciendo reabsorción (Bishara), pero luego de que el diente que impacta es reposicionado y el tratamiento ortodóntico se completa, la reabsorción radicular puede cesar (Cooke \& Wang). En nuestro caso, la radiografía pretratamiento muestra una sobreproyección del canino y su saco dentario con la zona apical del incisivo lateral, lo cual nos hace pensar en la existencia de reabsorción radicular. Sin em- bargo, a los 40 días de tratamiento el control radiográfico evidenció la desproyección de estas estructuras observando el tercio radicular apical contínuo junto a un espacio periapical normal.

Si bien pudimos realizar un manejo quirúrgoortodóntico adecuado, esto no es posible en todo los casos. Torres-Lagares et al. (2006), presentan un caso similar que presentaba la impactación del canino inferior izquierdo, donde se observaba un canino en posición horizontal bajo los ápices de los premolares. Ante esto, se prefirió indicar la extracción del mismo, puesto que al movilizarlo, este podría acercarse al foramen mentoniano, con el riesgo de la irritación del nervio alveolar inferior; por tanto la relación del diente con las estructuras anatómicas es un factor importante a considerar. Flores-Ruiz et al. (2007), reportan el caso de una paciente adulta con un canino superior impactado entre las raíces del incisivo lateral y central, con el desarrollo radicular y del foramen apical completo. Tras valorar las distintas opciones terapéuticas, se realizó un plan de tratamiento quirúrgicoortodóncico-protésico. Se instauró un tratamiento ortodóncico para generar el espacio de reposición del canino superior y luego se realizó la extracción del canino impactado junto con un tratamiento combinado de regeneración ósea e inserción inmediata de un implante, finalizando con la restauración protésica mediante una corona implantosoportada.

En nuestro caso, el tratamiento quirúrgicoortodóncico fue de elección ya que no existía el riesgo de comprometer elementos neurovasculares importantes o los dientes circundantes, por lo que no se contempló la extracción quirúrgica. Es importante tener en cuenta que esta puede ser realizada cuando no se pueda llevar a cabo la tracción ortodóncica, exista patología asociada a la inclusión (quistes voluminosos, infección, rizalisis, necrosis pulpar) o una enfermedad sistémica grave (Macias-Escalada et al.).

Debido a que es más frecuente que los caninos se desplacen hacia palatino, se les ha dado más atención en la literatura respecto de los dientes desplazados a vestibular en términos de su tratamiento quirúrgico. Se ha enfatizado en la complejidad de manejar dientes desplazados hacia vestibular sin que ocurran efectos adversos periodontales (Vanarsdall \& Corn), por lo que es fundamental un cuidadosa técnica quirúrgica, una colocación de encía marginal, el control de la inflamación, la ausencia de una fuerza excesiva y la adherencia gingival. La evidencia actual no ha logrado diferenciar que la técnica abierta es diferente 
BELTRÁN, V. V. J.; FLORES, B. P.; GARCíA, A. N.; CANTíN, M. \& FUENTES, F. R. Abordaje quirúrgico de canino maxilar incluido en posición vestibular para tracción ortodóncica: reporte de caso y revisión de la literatura. Int. J. Odontostomat., 5(3):220-226, 2011.

de un injerto posicionado apicalmente, es decir, erupción abierta, frente a la erupción cerrada, aunque existen informes contradictorios sobre el tejido queratinizado posicionado apicalmente y la técnica erupción cerrada utilizada para descubrir los dientes posición ectópica (Vermette et al.; Schubert \& Baumert, 2009). Nuestro caso es comparable a lo reportado por (Vanarsdall \& Corn).

Algunos autores han considerado que la técnica de reposición completa es preferible para los dientes impactados vestibulares debido a que sus resultados son más estéticos, ya que se obtienen menos cicatrices gingivales, menos longitud de la corona clínica y la reducción de la recidiva vertical en comparación con los injertos posicionados apicalmente (Vermette et al.). Vanarsdall \& Corn, han considerado el injerto posicionado apicalmente como sinónimo de técnica radical abierta, aunque el manejo de los tejidos alrededor de la corona anatómica, la conserva- ción de tejidos, y la colocación son fundamentales para un resultado periodontal y estético óptimo (Vanarsdall \& Corn; Vanarsdall, 2010).

El manejo de los caninos maxilares impactados es importante en términos de estética y función. Este procedimiento permite por lo general a los caninos una erupción correctamente en el arco dental, siempre y cuando se disponga de suficiente espacio para reposicionarlos, haciendo necesario en los casos indicados un correcto manejo quirúrgico-ortodóncico. En cuanto al tipo de cirugía, el pronóstico está condicionado tanto por la posición vertical del canino impactado como por la calidad del tejido queratinizado. Por consiguiente, un colgajo desplazado apical será más favorable si la corona del canino se encuentra más cerca del plano oclusal; en condiciones coronarias más altas, el colgajo de espesor total o técnica cerrada será el de elección, variando su diseño según la calidad del tejido queratinizado.

BELTRÁN, V. V. J.; FLORES, B. P.; GARCÍA, A. N.; CANTÍN, M. \& FUENTES, F. R. Surgical approach of vestibular position impacted maxillary canine for orthodontic traction: case report and literature review. Int. J. Odontostomat., 5(3):220226, 2011.

ABSTRACT: Impacted teeth present many problems. They can compromise tooth movement, esthetics, and functional outcomes. After the maxillary third molar, the maxillary canines are the most commonly encountered impaction, which can be impacted buccally or palatally, and are more common in female patients than in male patients. With a well-managed surgical and orthodontic treatment, impacted maxillary canines can be erupted and guided to an appropriate location in the dental arch. This article presents a surgical-orthodontic case of maxillary canine impacted buccally in an adult woman, treated with the closed technique for preservation of papillae.

KEY WORDS: impacted maxillary canine, surgical technique, orthodontic technique.

\section{REFERENCIAS BIBLIOGRÁFICAS}

Baccetti, T. A controlled study of associated dental anomalies. Angle Orthod., 68(3):267-74, 1998.

Becker, A. The Orthodontic Treatment of Impacted Teeth. 2nd ed. Abingdon, Informa Healthcare, 2007.

Bedoya, M. M. \& Park, J. H. A review of the diagnosis and management of impacted maxillary canines. J. Am. Dent. Assoc., 140:1485-93, 2009.

Bishara, S. E. Impacted maxillary canines: a review. Am. J. Orthod. Dentofacial. Orthop., 101(2):159-71, 1992.

Caccioli, P.; Bodini, G. \& Fiamminghi, L. Retained canine. Surgical exposure and guided traction. Dent. Cadmos, 58(9):54-61, 1990.

Cooke, J. \& Wang, H. L. Canine impactions: incidence and management. Int. J. Periodontics Restorative Dent., 26:483-91, 2006.

Ericson, S. \& Kurol, J. Early treatment of palatally erupting maxillary canines by extraction of the primary canines. Eur. J. Orthod., 10(4):283-95, 1988.

Elefteriadis, J. N. \& Athanasiou, A. E. Evaluation of impacted canines by means of computerized tomography. Int. J. Adult. Orthodon. Orthognath. Surg., 11(3):257-64, 1996.

Flores-Ruiz, R.; Infante-Cossío, P.; Llamas-Carreras, J. M.; Martínez-de-Fuentes, R.; Magallanes Abad, N. \& Gutiérrez-Pérez, J. L. Inserción de un implante simultáneo a la extracción de un canino superior incluido. Avances en Periodoncia, 19(2):85-9, 2007.

Jacobs, S. G. Reducing the incidence of palatally impacted 
maxillary canines by extraction of deciduous canines: a useful preventive/interceptive orthodontic procedure: case reports. Aust. Dent. J., 37(1):6-11, 1992.

Jacobs, S. G. The impacted maxillary canine. Further observations on aetiology, radiographics localization, prevention/interception of impaction, and when to suspect impaction. Aust. Dent. J., 41(5):310-6, 1996.

Jacoby, $\mathrm{H}$. The etiology of maxillary canine impactions. Am. J. Orthod., 84(2):125-32, 1983.

Kokich, V. G. Surgical and orthodontic management of impacted maxillary canines. Am. J. Orthod. Dentofacial Orthop., 126(3):278-83, 2004.

Leonardi, M.; Armi, P.; Franchi, L. \& Baccetti, T. Two interceptive approaches to palatally displaced canines: a prospective longitudinal study. Angle Orthod., 74(5):581-6, 2004.

Leonardi, R.; Liccardello, V.; Greco, M.; Rossetti, B. \& Barbato, $\mathrm{E}$. Alignment of a buccaly displaced maxillary canine in the late mixed dentition with a modified utility arch: a patient report. Word J. Orthod., 11(2):185-90, 2010.

Macias-Escalada, E.; Cobo-Plana, J.; Carlos-Villafranca, F. \& Pardo-López, B. Abordaje ortodóncico quirúrgico de las inclusiones dentarias. RCOE, 10(1):69-82, 2005

Magheri, P.; Cambi, S. \& Grandini, R. Restorative alternatives for the treatment of an impacted canine: surgical and prosthetic considerations. Pract. Proced. Aesthet. Dent., 14(8):659-64, 2002.

Marks, Jr. S. C.; Schroeder, H. E. \&Andreasen, J. O. Theories and mechanisms of tooth eruption. In: Andreasen, J. O.; Petersen, J. K. \& Laskin, D. M. (Eds.). Textbook and color atlas of tooth impactions. Diagnosis, treatment, and prevention. Copenhagen, Munksgaard, 1997.

Mcsherry, P. F. The ectopic maxillary canine: A review. Br. J. Orthod., 25(3):209-16, 1998.

Mochizuki, K.; Tsujino, K.; Yonezu, T. \& Yakushiji, M. The horizontally impacted situated in a labial position. Bull. Tokio Dent. Coll., 40(4):203-8, 1999.

Ngan, P.; Hornbrook, R. \& Weaver, B. Early timely management of ectopically erupting maxillary canines. Semin. Orthod., 11(3):152-63, 2005.

Peck, S.; Peck, L. \& Kataja, M. The palatally displaced canine as a dental anomaly of genetic origin. Angle Orthod., 64(4):249-56, 1994.

Pérez, M.; Pérez, P. \& Fierro, C. Alteraciones en la Erupción de Caninos Permanentes. Int. J. Morphol., 27(1):139-43, 2009.
Proffit, W. R.; Fields, H. W. \& Sarver, D. M. Contemporary Orthodontics. $4^{\text {th }}$ ed. Mosby, St. Louis, 2007.

Rusu, M. C.; Comes, C. A.; Stanciu, D.; Ciuluvica RC, Motoc A, Niculescu MC, \& Jianu AM. Altered anatomy in a case with a buccally impacted maxillary canine tooth. Rom. J. Morphol. Embryol., 51(4):783-6, 2010.

Schubert, M. \& Baumert, U. Alignment of impacted maxillary canines: critical analysis of eruption path and treatment time. J. Orofac. Orthop., 70(3):200-12, 2009.

Torres-Lagares, D.; Flores-Ruiz, R.; Infante-Cossío, P.; García-Calderón, M. \& Gutiérrez-Pérez, J. L. Transmigration of impacted lower canine. Case report and review of literature. Med. Oral Patol. Oral Cir. Bucal, 11(2):E171-4, 2006.

Vanarsdall, R. L. Efficient management of unerupted teeth: A time-tested treatment modality. Semin. Orthod. 16(3):212-21, 2010.

Vanarsdall, R. L. \& Corn, H. Soft-tissue management of labially positioned unerupted teeth. Am. J. Orthod., 72(1):53-64, 1977.

Vermette, M. E.; Kokich, V. G. \& Kennedy, D. B. Uncovering labially impacted teeth: apically positioned flap and closed-eruption techniques. Angle Orthod., 65(1):23-33, 1995.

Yavuz, M. S.; Aras, M. H.; Büyükkurt, M. C. \& Tozoglu, S. Impacted mandibular canines. J. Contemp. Dent. Pract., 8(7):78-85, 2007.

Zasciurinskiene, E.; Bjerklin, K.; Smailiene, D.; Sidlauskas, A. \& Puisys, A. Initial vertical and horizontal position of palatally impacted maxillary canine and effect on periodontal status following surgical-orthodontic treatment. Angle Orthod.; 78(2):275-80, 2008.

Dirección para correspondencia:

Dr. Víctor Javier Beltrán Varas

Dpto. Odontología Integral

Facultad de Medicina

Universidad de La Frontera

Calle Claro Solar No 115 - Of. 420

Temuco

CHILE

Email: vbeltran@ufro.cl

Recibido : 12-10-2011

Aceptado: 01-11-2011 